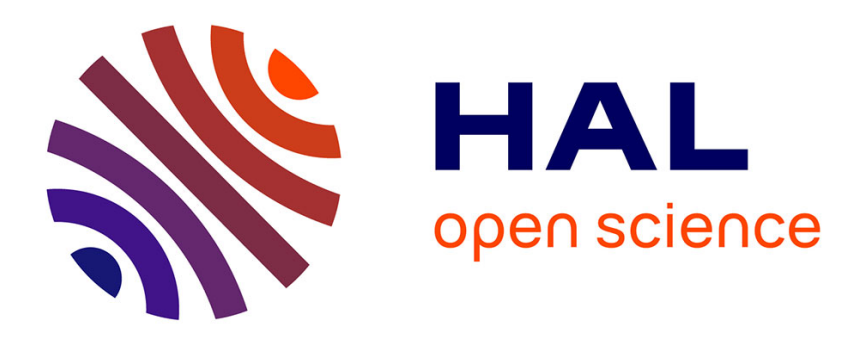

\title{
Interaction alumine plasma dans le Tokamak PETULA Groupe Petula
}

\section{To cite this version:}

Groupe Petula. Interaction alumine plasma dans le Tokamak PETULA. Revue de Physique Appliquée, 1977, 12 (6), pp.955-960. 10.1051/rphysap:01977001206095500 . jpa-00244266

\section{HAL Id: jpa-00244266 https://hal.science/jpa-00244266}

Submitted on 1 Jan 1977

HAL is a multi-disciplinary open access archive for the deposit and dissemination of scientific research documents, whether they are published or not. The documents may come from teaching and research institutions in France or abroad, or from public or private research centers.
L'archive ouverte pluridisciplinaire HAL, est destinée au dépôt et à la diffusion de documents scientifiques de niveau recherche, publiés ou non, émanant des établissements d'enseignement et de recherche français ou étrangers, des laboratoires publics ou privés. 


\author{
Classification \\ Physics Abstracts \\ $6.560-9.130-7.229-0.680$
}

\title{
INTERACTION ALUMINE PLASMA DANS LE TOKAMAK PETULA (*)
}

\author{
GROUPE PETULA (**) \\ Association EURATOM-CEA, Département de Physique du Plasma et de la Fusion Contrôlée \\ Service IGn, Centre d'Etudes Nucléaires, 85 X, 38041 Grenoble Cedex, France
}

(Reçu le 30 décembre 1976, accepté le 17 mars 1977)

\begin{abstract}
Résumé. - En vue de la construction de la chambre à vide en alumine de PETULA, on a mesuré les propriétés de dégazage de l'alumine et étudié l'interaction d'un plasma avec ce matériau. Des taux de dégazage de $10^{-11}$ à $10^{-12}$ torr $1 / \mathrm{s} \mathrm{cm}^{2}$ ont été atteints après étuvage à $150-200^{\circ} \mathrm{C}$. Les interactions alumine plasma ont été étudiées de deux manières différentes. Dans un cas - expérience ELISA - on a montré qu'avec des plasmas denses $\left(10^{13} \mathrm{e} / \mathrm{cm}^{3}\right)$ et froids (quelques $\mathrm{eV}$ ), la densité d'impureté était inférieure à $1 \%$. Pour des plasmas denses et chauds, la comparaison entre des décharges tokamaks obtenues avec des diaphragmes en tungstène ou en alumine, a montré que les caractéristiques du plasma sont meilleures dans le cas de l'alumine : les pertes et la contamination par l'oxygène sont plus faibles.
\end{abstract}

Abstract. - In connection with the construction of an alumina vacuum vessel on the PETULA Tokamak, measurements of the outgassing properties of alumina and studies of alumina-plasma interaction have been undertaken. Outgassing rates of $10^{-11}$ to $10^{-12}$ torr $1 / \mathrm{s} \mathrm{cm}^{2}$ are achieved for baking temperatures ranging between $150^{\circ}$ and $200^{\circ} \mathrm{C}$. Two categories of results have been obtained from the studies of alumina-plasma interaction depending on the plasma interacting with the alumina. For dense and cold plasma $n_{\mathrm{e}} \sim 10^{13}, T_{\mathrm{e}} \sim$ few eV, impurity concentrations lower than $1 \%$ are measured - ELISA experiment. For dense and hot plasmas, comparison between discharges produced with tungsten and alumina limiters shows an improvement of plasma characteristics in the case of alumina : ohmic power and oxygen contamination are significantly reduced.

1. Introduction. - Pour permettre la pénétration dans le plasma des champs électromagnétiques nécessaires au chauffage par T. T. M. P., la chambre à vide métallique utilisée précédemment sur le Tokamak PETULA a été remplacée par une nouvelle chambre à vide comprenant six secteurs isolants en alumine. Ces secteurs représentent une partie importante de la surface de l'enceinte et de ce fait les propriétés de dégazage de l'alumine peuvent jouer un rôle essentiel sur le vide de base de la machine et sur le taux de contamination de la décharge. Pour préciser le comportement de ce matériau, plusieurs mesures ont été entreprises :

- D'une part des mesures de gaz résiduel, de taux de dégazage en fonction de la température d'étuvage pour fixer les conditions de fonctionnement de la machine. Ces mesures ont été faites sur un sixième prototype de l'enceinte à vide et sur une enceinte cylindrique en alumine : expérience ELISA.

$\left(^{*}\right)$ Communication présentée au Congrès National de Physique des Plasmas, Paris, 6-10 décembre 1976.

(**) GROUPE PETULA : R. Bardet, M. Bernard, G. Briffod, M. Clement, R. Frank, A. Gauthier, M. Grégoire, P. Grelot, M. Hesse, G. Lamboley, F. Parlange, D. Pinet, G. Rey, B. Taquet, J. Weisse.
- D'autre part des mesures de taux d'impuretés du plasma produit soit dans l'enceinte ELISA soit dans le Tokamak PETULA avec la chambre à vide métallique mais équipé d'un diaphragme en alumine.

2. Le Tokamak PETULA : chambre à vide en alumine. - La chambre à vide de PETULA a un grand rayon de $72 \mathrm{~cm}$. Elle est constituée de six secteurs en alumine réunis à six queusots d'observation par des soufflets métalliques.

L'alumine utilisée est obtenue par pressage isostatique puis frittage à $1600^{\circ} \mathrm{C}$. Sa pureté est très élevée $(99,7 \%)$ et sa densité comprise entre 3,85 et 3,92. Sa porosité apparente est nulle. Après usinage cette alumine est chauffée dans l'air à $1100^{\circ} \mathrm{C}$ plusieurs heures pour brûler toute trace de graisse ou de métal présent sur sa surface.

Les secteurs de diamètre $\varnothing 340 \mathrm{~mm}$ font un angle de $40^{\circ}$ (Fig. 1). Compte tenu de leurs dimensions ils sont faits de deux éléments de $20^{\circ}$ usinés en forme toroïdale et assemblés au moyen d'un scellement verre à haute température [1]. La liaison alumine soufflets est obtenue à l'aide d'un double joint viton équipé d'un pompage auxiliaire. Tous les autres joints sont réalisés par soudure.

Les soufflets sont protégés du plasma par des 


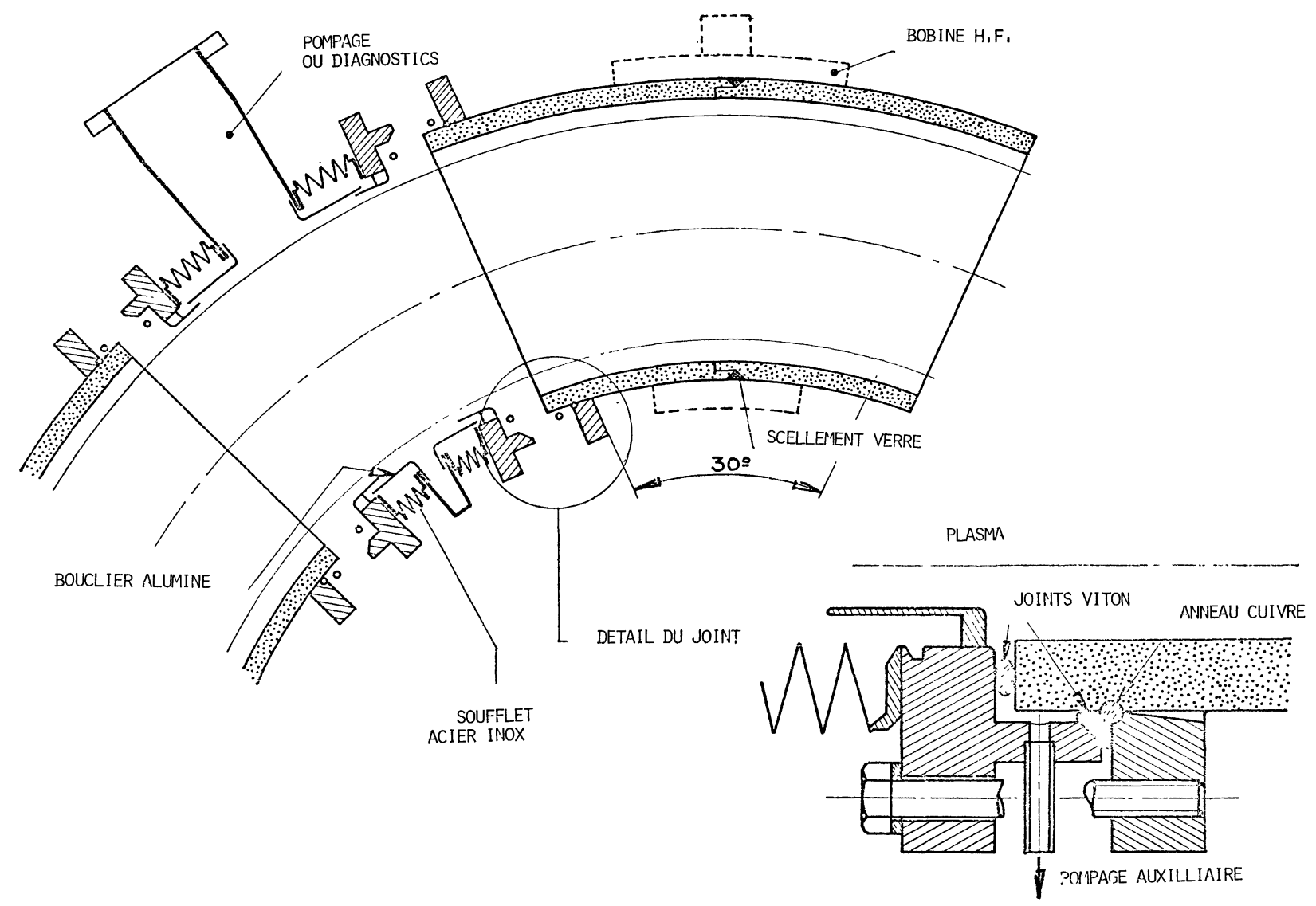

FIG. 1. - Coupe d'un sixième de la chambre à vide de PETULA.

[Cross section of one sixth of the PETULA vacuum chamber.]

boucliers en aluminium oxydé par électrolyse sur une épaisseur de $30 \mu$.

Au total $80 \%$ de la surface en regard du plasma est constituée d'alumine.

3. Vide statique. - Pour tester la solution technologique retenue pour la réalisation de la chambre à vide et estimer la valeur et la qualité de vide sur la machine, des essais ont été entrepris sur un sixième prototype de l'enceinte. Ce sixième est équipé d'une pompe turbomoléculaire type Pfeffer de $260 \mathrm{l} / \mathrm{s}$ de vitesse de pompage. Le gaz résiduel est analysé à l'aide d'un spectromètre de masse quadrupolaire et la mesure du taux de dégazage est faite en fermant la vanne reliant l'enceinte au groupe de pompage et en enregistrant l'augmentation temporelle de la pression totale. Le tableau I donne la valeur de la pression limite, du taux de dégazage et la composition du gaz résiduel en fonction de la température d'étuvage et de sa durée.

Il ressort de ces résultats que pour des températures d'étuvage supérieures à $150^{\circ}$, les taux de dégazage mesurés sont bas $\tau<10^{-11}$ torr $1 / \mathrm{s} \mathrm{cm}^{2}$. Ces résultats sont tout à fait comparables à ceux obtenus dans les mêmes conditions sur le sixième prototype de l'enceinte à vide en acier inoxydable $316 \mathrm{~L}$ utilisée précédemment sur l'expérience PETULA : $\theta=200^{\circ} \mathrm{C}$, $p=4,5 \times 10^{-9}, \tau=8 \times 10^{-12} \mathrm{~T} 1 / \mathrm{s} \mathrm{cm}^{2}$. L'analyse

\section{TABLEAU I}

Température d'étuvage

Durée

Pression de base (torr)

Taux de dégazage (T $1 / \mathrm{s} \mathrm{cm}^{2}$ )

$\mathrm{GaS}$ résiduel \%

$$
\begin{aligned}
& m=2 \\
& m=16 \\
& m=18 \\
& m=28 \\
& m=44
\end{aligned}
$$

\section{$20^{\circ}$}

$$
50 \mathrm{~h}
$$

$4 \times 10^{-7}$

$10^{-9}$

$130^{\circ}$

$60 \mathrm{~h}$

$10^{-8}$

$3 \times 10^{-10}$ $150^{\circ}$

$5 \times 10^{-9}$

$5 \times 10^{-11}$ $150^{\circ}$

$150 \mathrm{~h}$

$3,4 \times 10^{-9}$

$2 \times 10^{-11}$ $160^{\circ}$

$30 \mathrm{~h}$

$4,2 \times 10^{-9}$

$2 \times 10^{-11}$

42

3

28

17

7 $200^{\circ}$

$2 \times 10^{-12}$ 

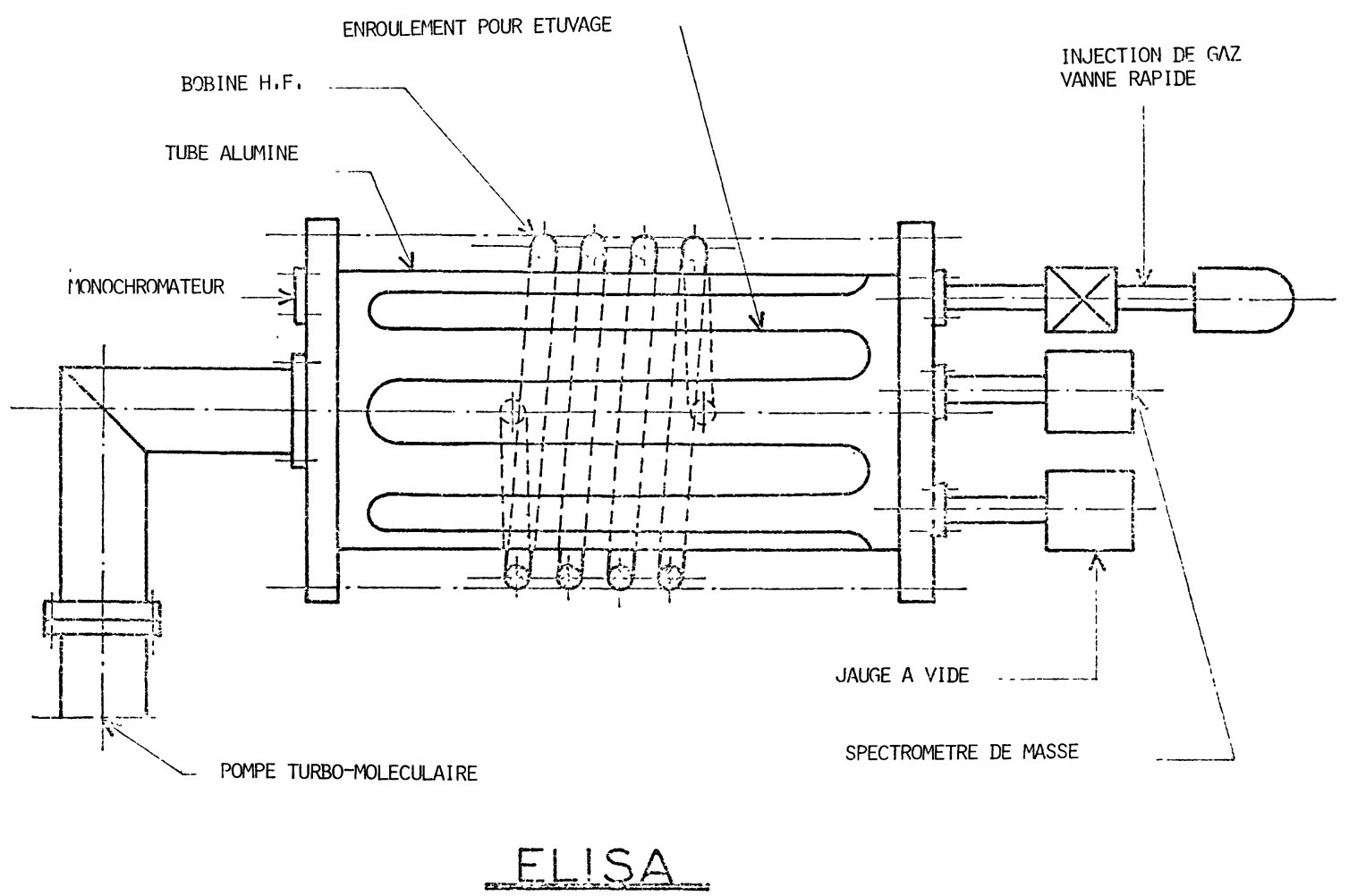

Fig. 2. - Montage expérimental ELISA.

[ELISA set up.]

de l'atmosphère résiduelle montre que celle-ci est constituée essentiellement d'hydrogène et du mélange $\mathrm{CO}+\mathrm{N}_{2}$ (masse 28).

Ces mêmes mesures faites sur chaque sixième définitif avant montage sur la machine confirment les valeurs ci-dessus. Les mesures sur l'enceinte complète sont en cours. Les premiers résultats après connection des diagnostics et un étuvage à $160^{\circ} \mathrm{C}$ pendant 1 semaine donnent un taux de dégazage de $5 \times 10^{-11} \mathrm{~T} 1 / \mathrm{s} \mathrm{cm}^{2}$ et le vide limite obtenu est de $1,310^{-8}$ torr.

4. Interaction faible. Plasma dense et froid. - Dans la phase initiale de la décharge Tokamak, les particules sont mal confinées et la paroi est en contact avec un plasma dense mais peu énergétique. Cette interaction a été simulée et étudiée sur l'expérience ELISA (Fig. 2). Les détails sur cette expérience ont été donnés par ailleurs [2, 3] nous ne rappellerons ici que les principaux éléments. L'enceinte à vide est un tube d'alumine cylindrique, $\varnothing=28 \mathrm{~cm}$, $L=54 \mathrm{~cm}$ relié à l'une de ses extrémités à une pompe turbomoléculaire $(260$ 1/s) et fermé à l'autre extrémité par une bride en acier inoxydable. L'étanchéité est faite par des joints or. L'enceinte est étuvable jusqu'à $250^{\circ} \mathrm{C}$. Le gaz est injecté à l'aide d'une vanne rapide. Lorsque la pression a atteint un état stationnaire, le gaz est ionisé à l'aide d'une bobine H. F. située à l'extérieur de l'enceinte. Cette bobine est alimentée soit par un oscillateur de $12 \mathrm{~kW}$ délivrant des impulsions longues $0-1$ s soit par un oscillateur de forte puissance $1 \mathrm{MW}$ durant $1 \mathrm{~ms}$. La fréquence de travail est de $200 \mathrm{kHz}$ et la fréquence de répétition 1 choc toutes les 4 minutes. Le plasma ainsi créé n'est pas confiné. Sa densité est déduite de la mesure de la raie $\mathrm{H}_{\beta}$ et l'évolution temporelle des composants du gaz est donnée par un spectromètre de masse quadrupolaire dont le temps de résolution sur ce montage est de $30 \mathrm{~ms}$.

Avec l'oscillateur à faible puissance $12 \mathrm{~kW}$ et une pression de $5 \times 10^{-3}$ torr d'hydrogène, la densité est de l'ordre de $10^{11} \mathrm{p} / \mathrm{cm}^{3}$ et la température électronique quelques $\mathrm{eV}$. Le tableau II donne la valeur des pressions partielles d'hydrogène et des principales

\section{TABLEAU II}

\section{Pressions partielles en torr}

$m / e$

Après 10 décharges

Après 60 décharges

Après étuvage à $200^{\circ} \mathrm{C}$
1216

$\begin{array}{ccc}1,5 \times 10^{-4} & 5 \times 10^{-3} & 7 \times 10^{-6} \\ 1,5 \times 10^{-4} & 5 \times 10^{-3} & 6 \times 10^{-6} \\ 1,5 \times 10^{-4} & 5 \times 10^{-3} & 5,5 \times 10^{-6}\end{array}$

$1,5 \times 10^{-4} 5 \times 10^{-3} 5,5 \times 10^{-6}$

$\begin{array}{ccc}18 & 28 & 44 \\ 10^{-5} & 1,5 \times 10^{-5} & 10^{-6} \\ 1,1 \times 10^{-6} & 5 \times 10^{-6} & 10^{-6} \\ 0 & 2,7 \times 10^{-6} & 10^{-6}\end{array}$

65

REVUE DE PHYSIQUe APPLIQUÉE. - T. 12, N 6, JUIN 1977 
TABLEAU III

$m / e$

Pression partielle après décharges

2

$5 \times 10^{-3}$

12

$10^{-6}$

impuretés de masse $16,18,28,44$. La première ligne de ce tableau correspond aux mesures faites au départ de l'expérience et sans conditionnement de l'enceinte. Le taux d'impuretés est de $6 \times 10^{-3}$ et il est indépendant de la pression de travail. L'impureté dominante est le pic 28. La seconde ligne du tableau correspond aux mêmes mesures faites après une série de 60 chocs.

Pour une même pression de travail, le taux d'impuretés a descendu à $2 \times 10^{-3}$. Cette diminution correspond à une réduction des pics 28 et 18 . Le même effet peut être obtenu par étuvage de l'enceinte. Les valeurs indiquées sur la troisième ligne du tableau montrent qu'après un étuvage à $220^{\circ} \mathrm{C}$, les pics 28 et 18 ont également diminué alors que les pics 16 et 44 n'ont pas varié.

Ceci nous permet d'espérer une amélioration sensible de la paroi d'alumine par des décharges de nettoyage à faible énergie.

Avec l'oscillateur à forte puissance ( $2 \mathrm{MW})$, et la même pression de remplissage d'hydrogène $5 \times 10^{-3}$ torr, le plasma produit est beaucoup plus dense $n>2 \times 10^{13} \mathrm{p} / \mathrm{cm}^{3}$ mais la température reste faible (quelques $\mathrm{eV}$ ). L'augmentation de densité ne modifie pas de façon notable, la nature et l'amplitude des pics d'impuretés. Les pics 28 et 16 restent les pics dominants et le taux d'impuretés est $1,3 \times 10^{-3}$ (Tableau III). L'absence dans le spectre des masses 14 et 32, suggère que les impuretés dominantes 28 et 16 sont le $\mathrm{CO}$ et le méthane.

Dans cette gamme de température et de densité qui recouvre les caractéristiques du plasma au tout début d'une décharge Tokamak, le taux d'impureté dû à l'interaction alumine plasma reste donc faible.

5. Interaction forte. Plasma dense et énergétique. Dans la première phase de l'exploitation de PETULA avec la chambre à vide métallique, trois types de diaphragme ont été utilisés: Tungstène, Alumine, Carbone. Des mesures détaillées des caractéristiques des décharges ont été publiées dans les Réfs. [4] et [5]. Dans ce qui suit on présente les principales différences entre les décharges dans le cas des diaphragmes Tungstène et Alumine. Comme le diaphragme reçoit une partie importante de l'énergie du plasma la comparaison entre les deux cas permet de préciser le comportement de l'alumine lorsqu'il est soumis au bombardement d'un plasma dense et énergétique.

5.1 Conditions eXPérimentales. - Dans les deux cas : diaphragme Tungstène et Alumine, le champ magnétique est 1,6 $\mathrm{T}$, le gaz utilisé est le deutérium, la chambre à vide est en acier inoxydable
316 L. Le diaphragme en Alumine (Fig. 3) est fait de 4 secteurs. Trois d'entre eux sont identiques, d'épaisseur $16 \mathrm{~mm}$. Le quatrième situé dans la partie extérieure du tore a une épaisseur de $75 \mathrm{~mm}$. Sa section a une forme trapézoïdale de façon à ce que le flux

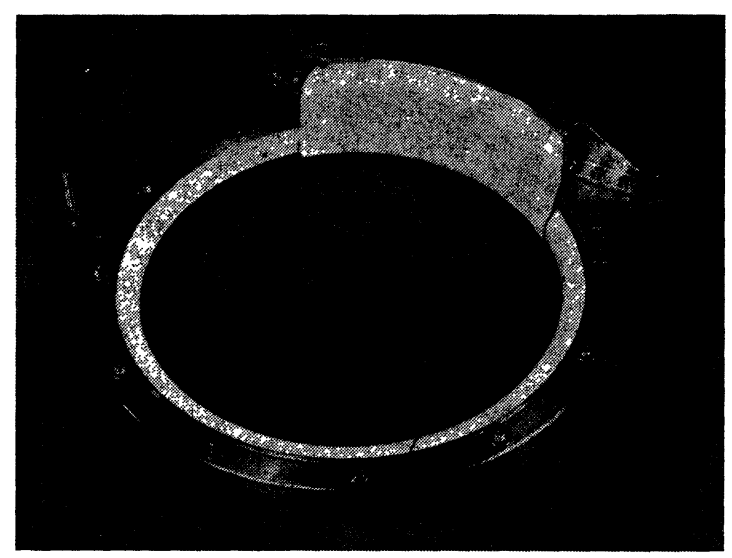

FIG. 3. - Photographie du diaphragme en alumine. [Photograph of the alumina limiter.]

de particules tombe sur la plus grande surface possible (Fig. 4). L'alumine est identique à celle utilisée pour les secteurs de la chambre à vide. Avant le montage le diaphragme est étuvé à $900^{\circ}$ pendant 20 heures dans l'air.

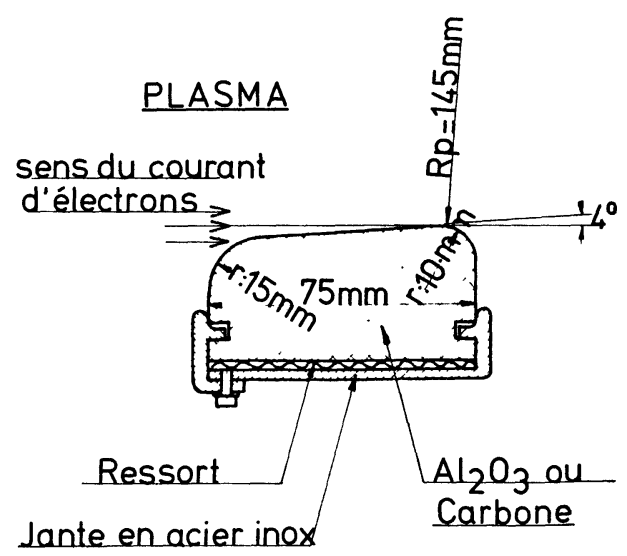

FIG. 4. - Section de la partie extérieure des diaphragmes $\mathrm{Al}_{2} \mathrm{O}_{3}$ ou carbone.

[Cross section of the external part of the alumina limiter.]

5.2 COMPARAISON DES DÉCHARGES. - Nous avons résumé dans le tableau IV les principales modifications des caractéristiques du plasma. Ces valeurs sont mesurées à $37 \mathrm{~ms}$, légèrement après le maximum de courant qui est de $70 \mathrm{kA}$. 


\begin{tabular}{lcc}
\multicolumn{3}{c}{ TableaU IV } \\
& $\begin{array}{c}\text { Diaphragme } \\
\text { Tungstène }\end{array}$ & $\begin{array}{c}\text { Diaphragme } \\
\text { Alumine }\end{array}$ \\
Tension par tour, volt & 4,6 & - \\
Puissance ohmique, $\mathrm{W} / \mathrm{cm}^{3}$ & 1,1 & 0,75 \\
Densité moyenne, $10^{13} \mathrm{~cm}^{-3}$ & 2,5 & 2,8 \\
$T_{\mathrm{e}}$ sur l'axe & 510 & 435 \\
$T_{\mathrm{i}}$ sur l'axe & 230 & 210 \\
Temps de vie de l'énergie & 1,9 & 2,9 \\
$Z_{\text {effectir }}$ & 6,3 & 3 \\
Densité d'impuretés & & \\
Oxygène, $10^{11} \mathrm{~cm}^{-3}$ & 30 & 8 \\
Carbone, $10^{11} \mathrm{~cm}^{-3}$ & 3 & 3
\end{tabular}

Le phénomène marquant est la diminution du taux d'oxygène dans la décharge dans le cas d'un diaphragme alumine. La densité d'oxygène est calculée à partir de l'intensité de la raie OVI $1023 \AA$ et dans les deux cas, elle est en accord avec les valeurs de $Z_{\text {efficace }}$ déduite des paramètres expérimentaux de la décharge.

Des analyses de gaz ont également été faites avec un spectromètre de masse immédiatement après un choc. Le pic prédominant correspond à la masse 28 comme dans ELISA et d'autres expériences similaires [6]. On constate que le taux d'impuretés passe de 0,25 à 0,08 (Figs. 5 et 6 ) quand on passe du diaphragme tungstène au diaphragme alumine.

Cette diminution du taux d'oxygène $a$ un effet positif sur le bilan d'énergie de la décharge : les pertes d'énergie rayonnée par les impuretés sont moindres. De fait, dans les deux cas, $\beta_{\text {poloidal a la }}$ même valeur 0,7 , mais dans les décharges avec diaphragme alumine la puissance ohmique nécessaire pour obtenir ce résultat est $30 \%$ plus faible ; c'est-à-

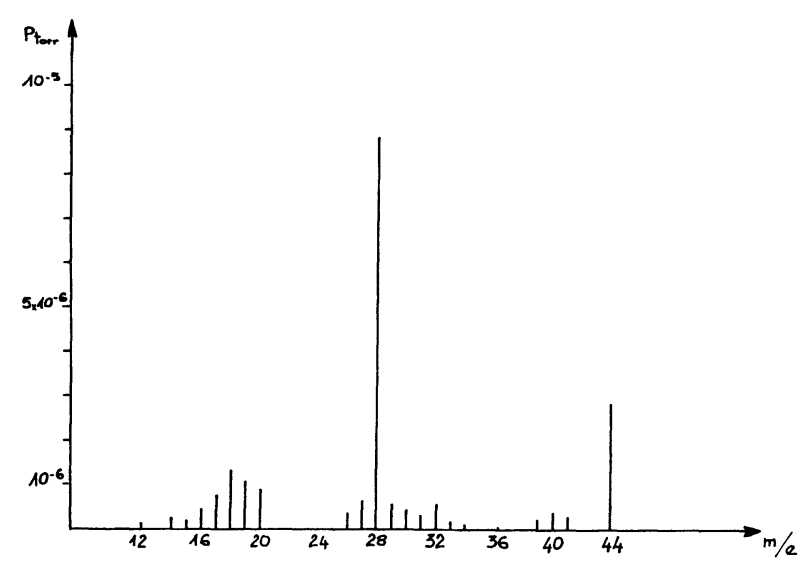

Fig. 5. - Spectre de masses du gaz résiduel après un choc Diaphragme W

Chocs 3513-3521

$$
\begin{aligned}
& I=72 \mathrm{kA} \quad n=2,7 \times 10^{13} \mathrm{~cm}^{-3} \\
& \Sigma P_{\text {impuretés }}=1,7 \times 10^{-5} \text { torr } \\
& P_{\mathrm{D}_{2}}=7 \times 10^{-5} \text { torr } .
\end{aligned}
$$

[Mass spectrogram of the residual gas after a shot with tungsten limiter.]

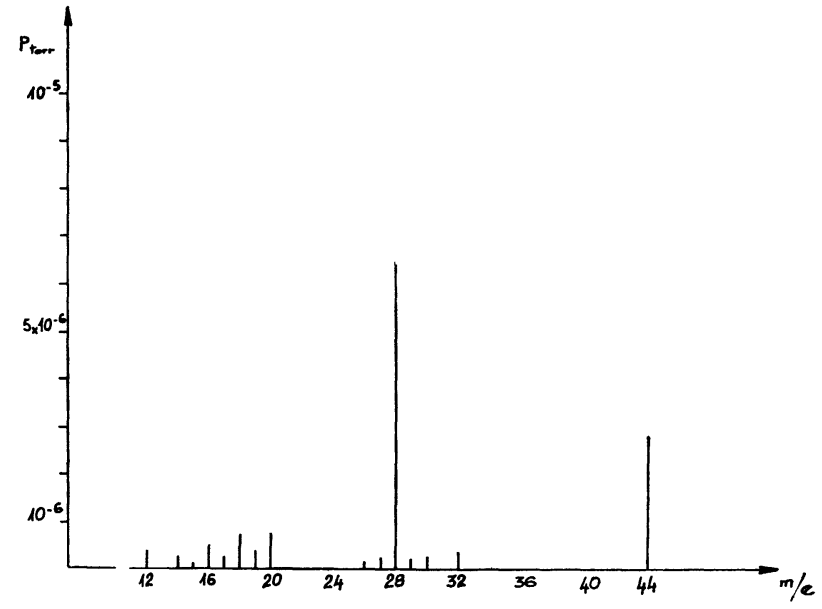

FIG. 6. - Spectre de masses du gaz résiduel après un choc avec diaphragme alumine.

Diaphragme Alumine

Chocs 4332-4357

$$
\begin{aligned}
& I=70 \mathrm{kA} \quad n=3,2 \times 10^{13} \mathrm{~cm}^{-3} \\
& \sum P_{\text {impuretés }}=1,2 \times 10^{-5} \text { torr } \\
& P_{\mathrm{D}_{2}}=1,4 \times 10^{-4} \text { torr }
\end{aligned}
$$

[The same as figure 5 for the alumina limiter.]

dire que le temps de vie de l'énergie augmente de $50 \%$ passant de 1,9 à $2,9 \mathrm{~ms}$.

5.3 Comportement du matériau. - Pour des conditions expérimentales comparables, le changement d'un diaphragme en tungstène pour un diaphragme en alumine se traduit par une diminution d'un facteur 4 de la densité d'oxygène. Ceci suggère que le diaphragme joue un rôle important dans la quantité d'oxygène présente durant la décharge. Une interprétation possible de cette différence peut être due à la présence d'oxyde de Tungstène à la surface du limiter. Le coefficient de pulvérisation de $\mathrm{WO}_{3}$ est un ordre de grandeur supérieur à celui de $\mathrm{Al}_{2} \mathrm{O}_{3}$ et cette différence croît avec la température [7].

D'autre part les deux oxydes sont sujets à la pulvé-. risation de l'oxygène. En fait après 1500 décharges le diaphragme alumine devient jaune sombre. L'analyse par fluorescence X montre qu'il ne s'agit pas de dépôt métallique mais d'un changement stœchiométrique. Sous l'effet du bombardement du plasma l'alumine perd une partie de son oxygène pour constituer un oxyde amorphe. En réchauffant le diaphragme à l'air à $600^{\circ} \mathrm{C}$ il retrouve sa composition antérieure et sa couleur.

Le principal inconvénient de l'alumine est sa faible résistance aux chocs thermiques. Après un peu plus d'un millier de décharges et plusieurs séries de décharges de nettoyage, le secteur extérieur du diaphragme montre des cassures (Fig. 7). Les décharges de nettoyage sont des décharges de $30 \mathrm{kA}-10 \mathrm{~ms}$ non confinées $(B=0,15$ tesla) avec un taux de répétition de $0,3 \mathrm{~Hz}$. Après $20 \mathrm{~min}$. d'opération la température du diaphragme mesurée dans la masse 


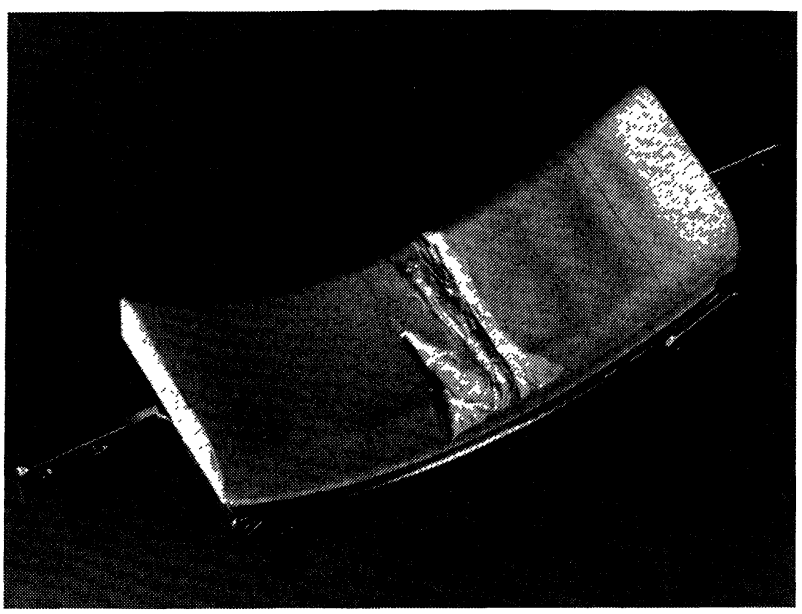

FIg. 7. - Photographie de la partie extérieure du diaphragme alumine après utilisation.

[External part of the alumina limiter after use.]

par des thermocouples atteint $200^{\circ} \mathrm{C}$ dans le secteur extérieur et $50^{\circ} \mathrm{C}$ dans le secteur intérieur. On peut donc penser que les cassures observées se sont pro-

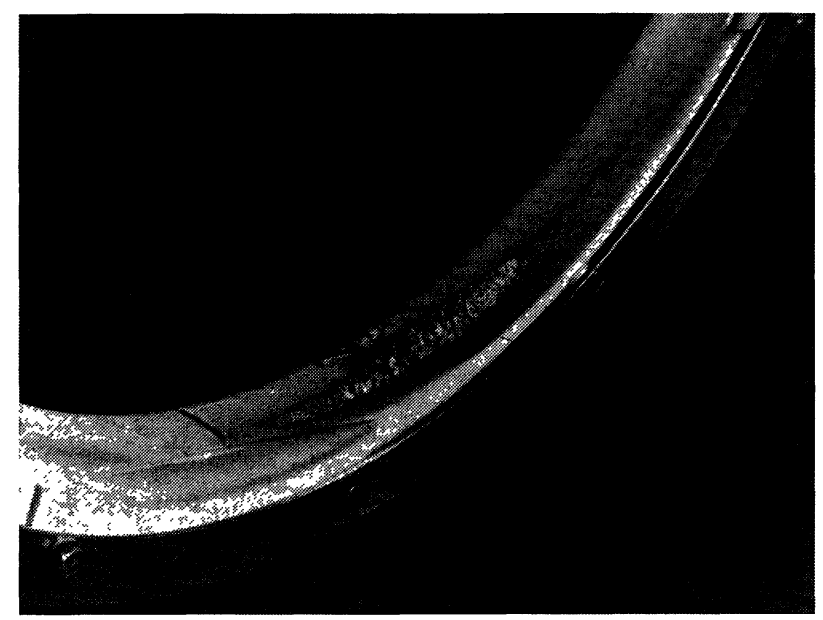

FIG. 8. - Photographie de la partie intérieure du diaphragme alumine après utilisation.

[Internal part of the alumina limiter after use.] duites au cours des décharges de nettoyage. En fait le secteur intérieur ne présente pas de cassures mais seulement des zones de fusion (Fig. 8). Cette fusion est très probablement due aux décharges normales à la fin desquelles le plasma vient en général mourir vers l'intérieur du tore.

Dans le voisinage immédiat du diaphragme, les parois de la chambre sont couvertes par une couche de $1 \mu \mathrm{m}$ constituée d'aluminium, à $20 \%$ sous forme métallique et à $80 \%$ sous forme d'oxyde.

Il faut souligner que le bombardement auquel a été soumis l'alumine dans cette expérience est beaucoup plus important que celui auquel ce matériau sera soumis dans la chambre en alumine. La puissance par $\mathrm{cm}^{2}$ reçue par le limiter est au moins 100 fois plus grande que celle reçue par les parois d'un Tokamak.

6. Conclusion. - De ces différents essais on peut tirer les conclusions suivantes :

- Un vide propre peut être obtenu dans une chambre en alumine dans des conditions similaires à celles des chambres en acier inoxydables.

- La quantité d'impuretés produite pendant les interactions avec un plasma peut être réduite par des décharges de nettoyage à faible énergie ou par étuvage.

- Les interactions plasma diaphragme jouent un rôle très important dans la contamination des décharges Tokamak.

- La contamination par l'oxygène en particulier baisse d'un facteur 4 lorsqu'on utilise un diaphragme en alumine au lieu d'un diaphragme en tungstène.

L'alumine, malgré cet avantage, peut difficilement être utilisée comme matériau pour le diaphragme car elle résiste mal aux chocs thermiques. Par contre ces propriétés montrent qu'elle peut être employée pour la construction de la chambre à vide car la charge thermique sur les parois est beaucoup plus faible que sur le diaphragme.

\section{Bibliographie}

[1] Monneraye, M., Scellement métal céramique, Revue technique Phillips, $\mathrm{N}^{\circ} 5$.

[2] Frank, R., Gauthier, A., Lambolley, G., Proceedings of Fifth Symposium on Engineering Problems, Princeton (1973) 394

[3] Frank, R., Le Vide 160 (1974) 314.

[4] Petula Group, The Petula Tokamak Experiment : effects of low $Z$ materials on plasma characteristics 6 th Int. Conf. on Plasma Physics and Controlled Nuclear Fusion, Berchtesgaden (1976).

[5] Petula Group, Influence of the limiter material on the plasma characteristics in the Petula Tokamak, Nucl. Fusion 16 (1976) 579.

[6] McCracken, G. M., Partridge, J. W., The Chemical Interaction of Hydrogen Discharges with non Metals, CULHAM LABORATORY, CLM-P 454 (1976).

[7] Kelly, R., NCHI Q. LAM, Radiat. Eff. 19 (1973) 39. 